$$
\begin{gathered}
C D+950472-12 \\
\text { UCRL-JC-119165 } \\
\text { PREPRINT }
\end{gathered}
$$

\title{
Infrared Hyperspectral Imaging Results from Vapor Plume Experiments
}

\author{
C. L. Bennett \\ M. R. Carter \\ D. J. Fields \\ F. D. Lee
}

This paper was prepared for submittal to the Society of Photo-Optical Instrumentation Engineers

Orlando, FL

April 17-21, 1995

April 17, 1995

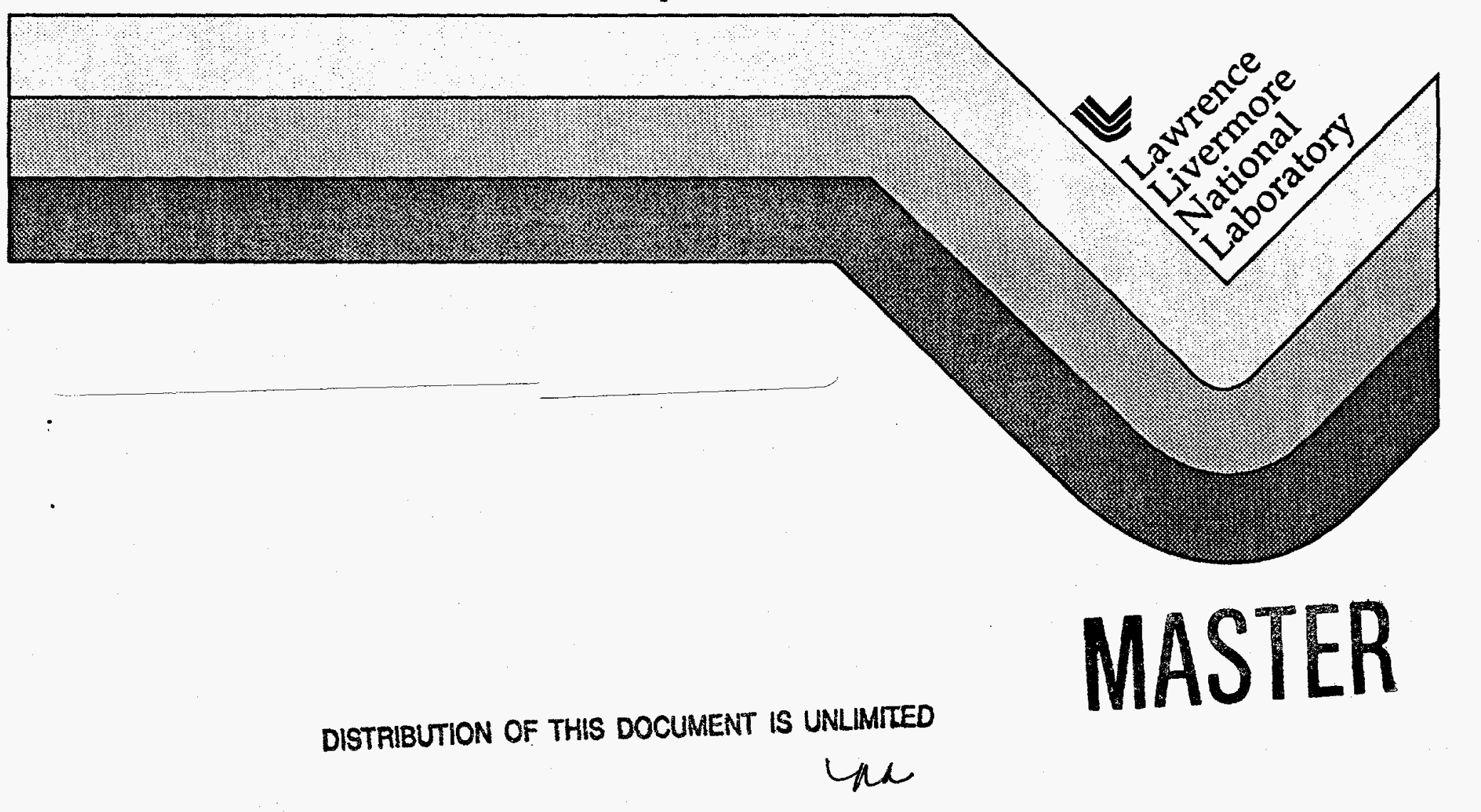




\section{DISCLAIMER}

This document was prepared as an account of work sponsored by an agency of the United States Government. Neither the United States Government nor the University of California nor any of their employees, makes any warranty, express or implied, or assumes any legal liability or responsibility for the accuracy, completeness, or usefulness of any information, apparatus, product, or process disclosed, or represents that its use would not infringe privately owned rights. Reference herein to any specific commercial product, process, or service by trade name, trademark, manufacturer, or otherwise, does not necessarily constitute or imply its endorsement, recommendation, or favoring by the United States Government or the University of California. The views and opinions of authors expressed herein do not necessarily state or reflect those of the United States Government or the University of California, and shall not be used for advertising or product endorsement purposes. 


\section{DISCLAIMER}

Portions of this document may be illegible in electronic image products. Images are produced from the best available original document. 


\title{
Infrared Hyperspectral Imaging Results from Vapor Plume Experiments
}

\author{
Charles L. Bennett, Michael R. Carter, David J. Fields, F. Dean Lee \\ Lawrence Livermore. National Laboratory \\ P.O. Box 808, Livermore, CA. 94551
}

\begin{abstract}
In this article, recent measurements made with LIFTIRS, the Livermore Imaging Fourier Transform InfraRed Spectrometer, are presented. The experience gained with this instrument has produced a variety of insights into the tradeoffs between signal to noise ratio (SNR), spectral resolution and temporal resolution for time multiplexed Fourier transform imaging spectrometers. This experience has also clarified the practical advantages and disadvantages of .Fourier transform hyperspectral imaging spectrometers regarding adaptation to varying measurement requirements on SNR vs. spectral resolution, spatial resolution and temporal resolution.
\end{abstract}

\section{INTRODUCTION}

Although a great deal of information about a scene can be deduced from shapes and forms apparent in a two dimensional image, spectral measurements add a third dimension. Multispectral imagers, characterized by having only a few spectral channels, such as the sensors on Landsat for example, enable qualitative identification of distinctive terrain features; such as vegetation stress, or mineral species. Hyperspectral imagers, characterized by having a large number of spectral channels, enable definitive identification and quantitative measurement of the composition of objects in the field of view. Infrared hyperspectral imagers are particularly useful for remote chemical analysis, since almost all molecules have characteristic rotationvibration spectra in the infrared, and a broad portion of the so-called "fingerprint" region of the infrared spectrum lies within the thermal infrared water window between 8 to $12 \mu \mathrm{m}$, or 750 to $1250 \mathrm{~cm}^{-1}$. An example of the nature of such a hyperspectral data cube is illustrated in figure 1.

Until recently, very few infrared hyperspectral imaging instruments were available. We have recently developed an infrared hyperspectral imager based on an imaging. Fourier transform spectrometer, and have begun to perform instrument development tests and field measurements: We have found the technique of imaging FTIR to be quite flexible in terms of the facility for tradeoff of spectral resolution for temporal resolution, with no effect on the degree of spatial resolution. The very high optical throughput characteristic of FT spectrometers in general enables high quality and high speed acquisition of imagery. Indeed, the interferometer need produce little more than an average reduction by $50 \%$ (due to the beam splitter) in the intensity of light which reaches the image focal plane. For this reason, it is possible to acquire thermal infrared hyperspectral imagery with an ambient temperature interferometer, which is in sharp contrast to the situation with dispersive imaging spectrometers. Furthermore, it is possible to use the infrared imagery itself to aid in pointing and tracking a given subject.

In this article, first a brief history of the development of imaging FTIR spectrometers will be given. Second, the choice of operating conditions is discussed, in particular the number of points measured in an interferogram, which directly determines the instrumental resolution. Third a series of measurements of gaseous plumes against both sky and hills will be discussed. Finally, the impact of temporal fluctuations on the derived spectra will be addressed.

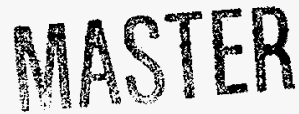




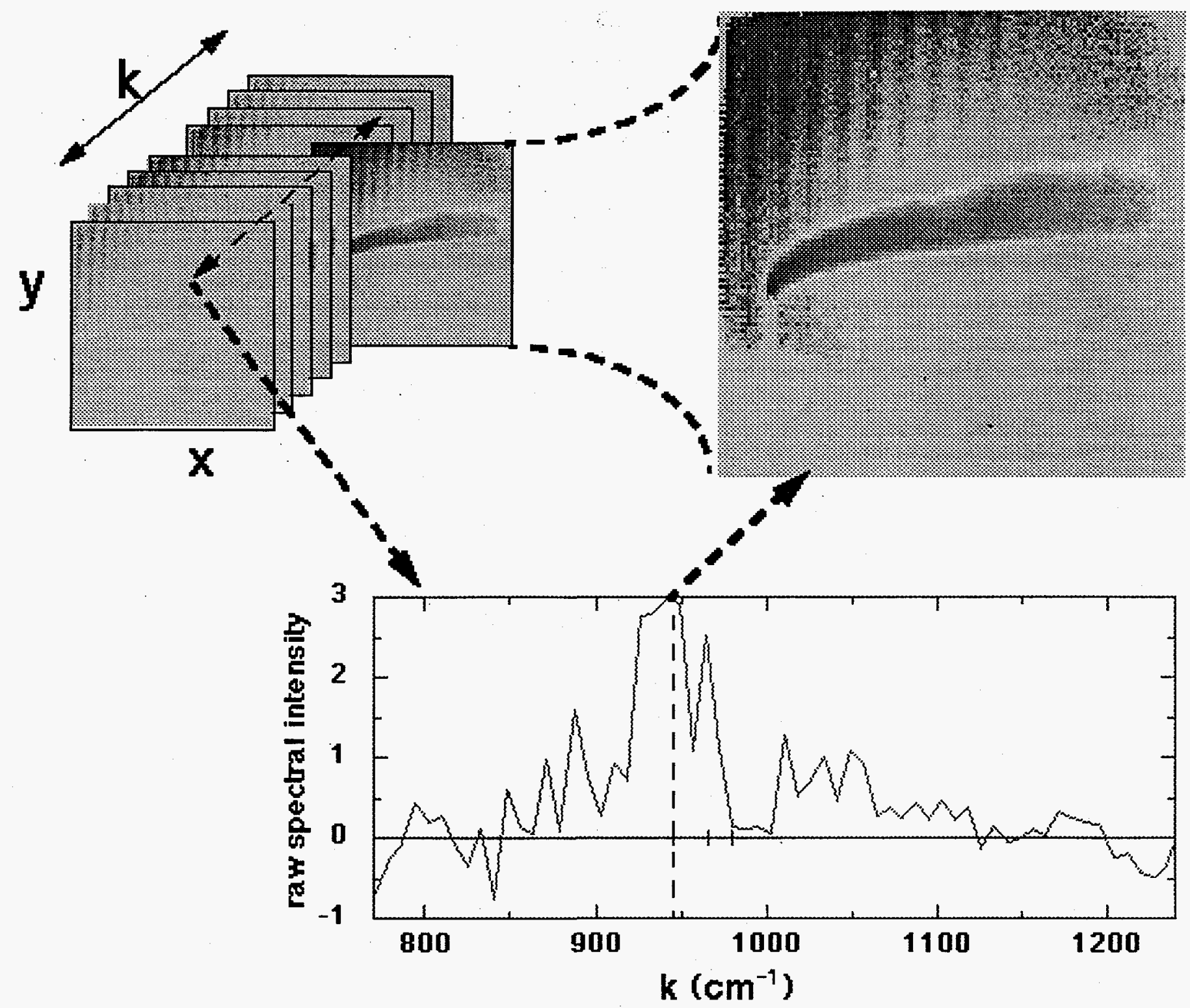

Figure 1. An illustration of hyperspectral imaging. A data cube, as indicated in the upper left of the diagram, consists of a series of images of a scene, each image corresponds to the appearance of the scene as viewed at a given wavenumber $k$, as is indicated by the arrow extending from the spectral plot to the image in the upper right. On the other hand, a line through the stack of frames in a data cube corresponds to the spectrum of light observed at the corresponding $(x, y)$ location in the image, as is indicated by the arrow piercing the data cube and the downward pointing arrow in the figure. 


\section{DEVELOPMENT HISTORY}

An imaging Fourier transform spectrometer was first described in a 1972 patent by A.E. Potter, $\mathrm{Jr}^{1}$. This concept was quite far ahead of its time then, however, and it was not until. 1980 that results from a working instrument, with a rather modest 42 element array in the focal plane, were published ${ }^{2}$. Truly "imaging" FTIR spectrometers required the development of relatively large format infrared focal plane array (FPA) detectors. Also, in order to handle the computational demands in a reasonable time, relatively inexpensive, fast computers were needed. As an example, an array of $128 \times 128$ pixels generates 16,384 separate interferograms, which must be Fourier transformed before a spectrum can be seen. With a single board computer, it is now possible to perform these Fourier transforms as quickly as they can be acquired.

In 1993, we obtained our first observations with the prototype LIFTIRS instrument. These results, and a general discussion of the advantages and disadvantages of imaging FT.IR spectrometers as compared to dispersive hyperspectral imagers were presented at the 1993 SPIE conference in Orlando, Florida ${ }^{3}$. Further results of gaseous effluent imaging and identification were presented at the 1993 SPIE conference in Innsbruck, Austria ${ }^{4}$. The LIFTIRS instrument has been significantly improved since these initial experiments, and detailed discussion of the operational characteristics are discussed by Carter, et al. 5 .

Spaceborne imaging FTIR spectrometers are discussed by Beer6, as well as the general theory of FTIR instruments, a comparison with dispersive spectrometers, and a treatment of the general problem of remote sensing through the atmosphere in the infrared portion of the spectrum.

\section{OPTIMIZING DATA ACQUISITION PARAMETERS}

Assuming a white noise spectrum, the relation between the signal to noise ratio measured in the interferogram, $\mathrm{SNR}_{\mathbf{x}}$, (which is determined by the rms noise level in the interferogram to the maximum swing in the interferogram at the zero phase difference point for a properly phase compensated interferometer) to the signal to noise ratio measured in the spectral domain, $\mathrm{SNR}_{\mathrm{k}}$, is

$$
S N R_{k}=S N R_{x} * \frac{\sqrt{N_{k}}}{m}
$$

where $N_{k}$ is the total number of spectral channels, which is half $N_{x}$, the number of interferogram samples for a one sided interferogram, and $m$ is the spectral bandpass of the system in units of channels. As an example, for the case $m=1$, only a single spectral component is present, and measuring $N_{X}$ points in the interferogram improves the signal to noise by a factor of $\sqrt{N_{X}}$ over that of a single measurement. The remaining $\sqrt{2}=\sqrt{N_{x} / N_{k}}$, factor is obtained because in FTIR, only the real part of the properly phase compensated Fourier transform contains signal, while the noise is evenly divided between real and imaginary components.

From the simple expression (1) it follows that for a fixed amount of time, the SNR per channel in the spectral domain is linearly proportional to the FWHM of the instrumental resolution, since the resolution is inversely proportional to $N_{x}$, the time required to acquire a single interferogram is linearly proportional to $N_{x}$, and the spectral bandpass is also proportional to $\mathrm{N}_{\mathrm{x}}$. For spectral features which are much narrower than the instrumental resolution, there is no improvement in the detection threshold obtained with better spectral resolution. On the other 
hand, for spectral features which are broad compared to the instrumental resolution, there is an improvement in the detection threshold with increasingly coarse resolution. For this reason, the optimum spectral resolution, in terms of achieving the lowest detection threshold, is obtained by choosing the broadest resolution which still enables separation of neighboring spectral features.

As a specific example, for the case in which a mixture of $\mathrm{SF}_{6}$ and $\mathrm{NH}_{3}$ is to be examined, an optimal choice of resolution is approximately $10 \mathrm{~cm}^{-1}$. The reason for this choice is based on the nature of the spectra of $\mathrm{SF}_{6}$ and $\mathrm{NH}_{3}$. The fully resolved spectrum of $\mathrm{NH}_{3}$ has line widths of approximately $0.1 \mathrm{~cm}^{-1}$, and is of course completely specific to ammonia. Observed at coarser resolution the finest features in the spectrum are not observed, however, there is still a very distinctive set of two spectral groups of nearly the same intensity, with widths of approximately 4 $\mathrm{cm}^{-1}$, located at approximately 930 and $966 \mathrm{~cm}^{-1}$. Similarly, when observed at less than the full resolution required to observe all of its fine structure, $\mathrm{SF}_{6}$ has a large broad group centered at about $945 \mathrm{~cm}^{-1}$, and extending over approximately $9 \mathrm{~cm}^{-1}$, and a much weaker group at somewhat lower wavenumber. In order to separate and quantify this particular pair of chemicals, the LIFTIRS instrument can be operated at a spectral resolution of about $8 \mathrm{~cm}^{-1}$, and a complete data cube can be taken in a period of $2.5 \mathrm{~s}$.

The number of distinct $8 \mathrm{~cm}^{-1}$ bands within the bandwidth between 750 and $1250 \mathrm{~cm}^{-1}$ is approximately 64 . However, if the two strongest peaks in a given molecular spectrum are observed, the number of distinct pairs of $8 \mathrm{~cm}^{-1}$ groups within the same bandwidth is $64 \cdot 63 / 2=$ 2016.

\section{FIELD DATA}

In a series of experiments conducted at the Nevada Test Site at the Spill Test Facility, various volatile compounds were released, with direct measurements of the source terms. For one of these cases, in which a mixture of $\mathrm{SF}_{6}$ and $\mathrm{NH}_{3}$ was released, portions of the infrared hyperspectral image data are displayed in figure 2. At the top of the figure, three single wavenumber image slices of the data cube are shown for wavenumbers which correspond to $\mathrm{SF}_{6}, \mathrm{NH}_{3}$, and a background spectral channel. A spectrum from a point within the plume is represented by the heavy line in the plot in figure 2. Reference spectra from $\mathrm{SF}_{6}$ and $\mathrm{NH}_{3}$ are indicated by the light gray lines. From the measured wind velocity field, it was possible to estimate the downwind concentration distribution, and from the observed shape and thickness of the plume, it was possible to estimate the plume thickness. At the position of the center of the fields of view displayed at the top of figure 2, the estimated integrated concentration length products were $3200 \mathrm{ppm} \cdot \mathrm{m}$ for $\mathrm{NH}_{3}$ and $190 \mathrm{ppm} \cdot \mathrm{m}$ for $\mathrm{SF}_{6}$. After a 15 minute purge of the gas release system, we attempted to take a background spectrum for reference. It surprised us to find that although there was indeed no $S_{6}$ present, there was a very distinct hyperspectral signature of $\mathrm{NH}_{3}$ still being emitted from the gas release manifold. The images at the wavenumbers corresponding to $\mathrm{SF}_{6}$ and $\mathrm{NH}_{3}$ after the 15 minute purge are displayed in the lower two images of figure 2. From the observed radiance, the integrated concentration length product at the position of the center of the image of the $\mathrm{NH}_{3}$ was found to be $30 \mathrm{ppm} \cdot \mathrm{m}$. Even at this lower thickness, it is well above detection threshold in the hyperspectral imaging data.

The visibility of the plumes remained high for quite a long distance downwind. Since the plume width tends to grow diffusively, and thus only increases as the square root of the downwind distance, the average concentration tends to decrease linearly with downwind distance. The observed radiance for an optically thin plume, however, depends on the integrated product of the concentration and plume thickness, and thus only decreases as the square root of the downwind distance once the plume has come into equilibrium with the ambient air temperature. 


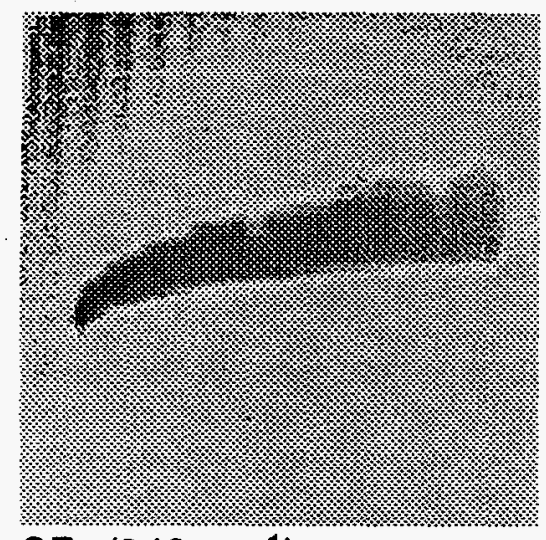

$\mathrm{SF}_{6}\left(946 \mathrm{~cm}^{-1}\right)$

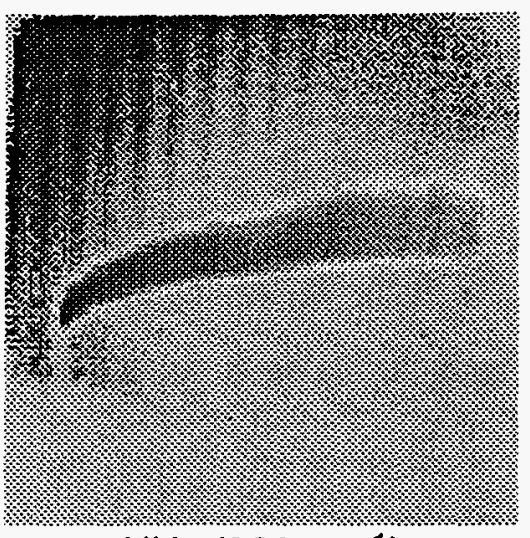

$\mathrm{NH}_{3}\left(966 \mathrm{~cm}^{-1}\right)$

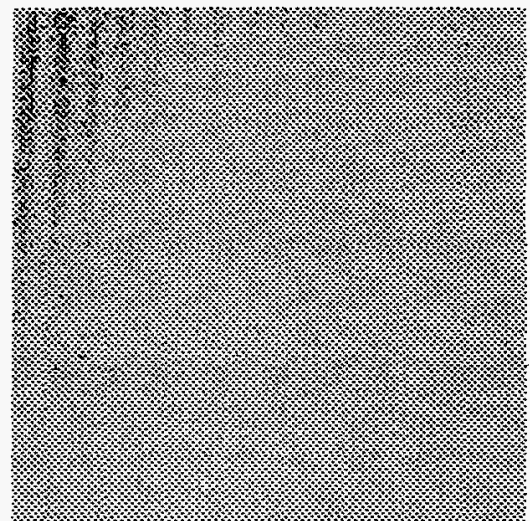

Background $\left(980 \mathrm{~cm}^{-1}\right)$

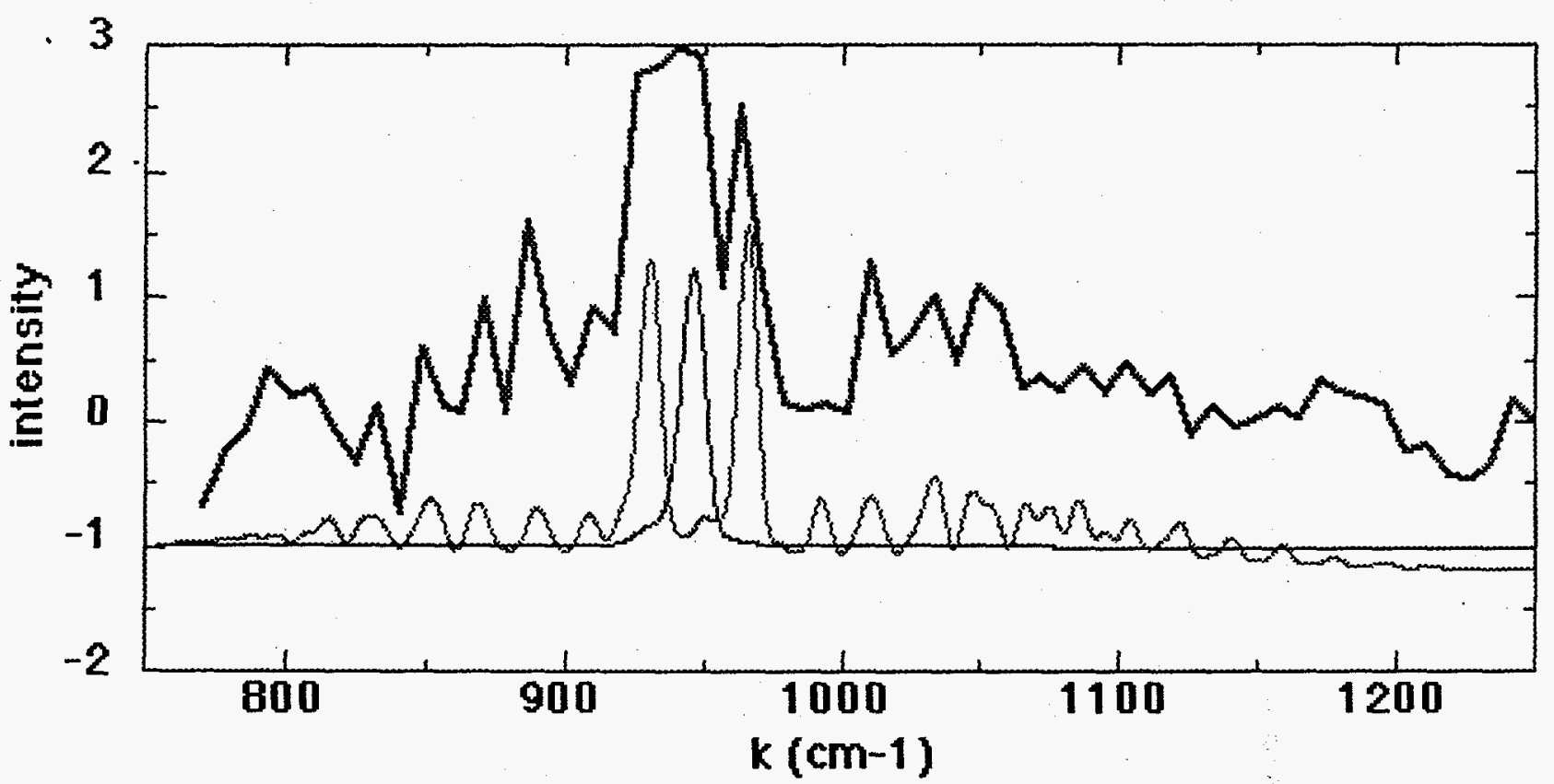

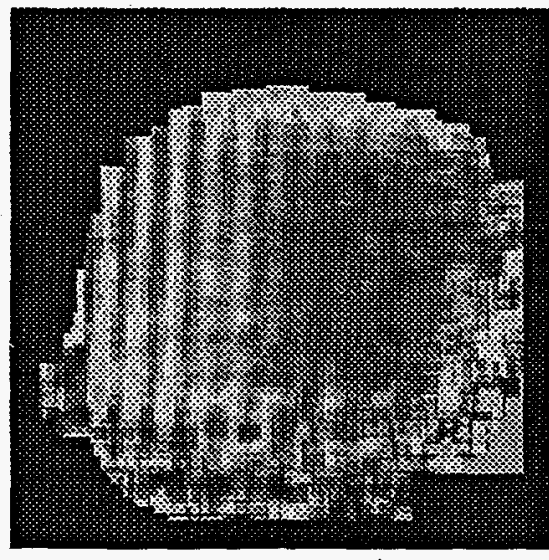

$\mathrm{SF}_{6}\left(946 \mathrm{~cm}^{-1}\right)$

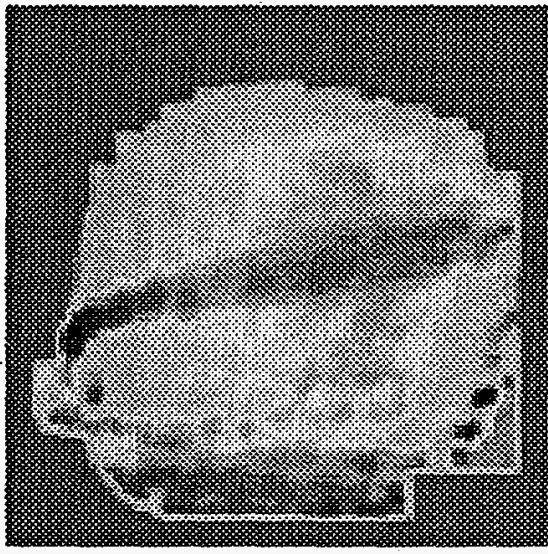

$\mathrm{NH}_{3}\left(966 \mathrm{~cm}^{-1}\right)$

After 15 minute purge

Figure 2. Observations of $\mathrm{SF}_{6}$ and $\mathrm{NH}_{3}$ with clear sky in the background. 


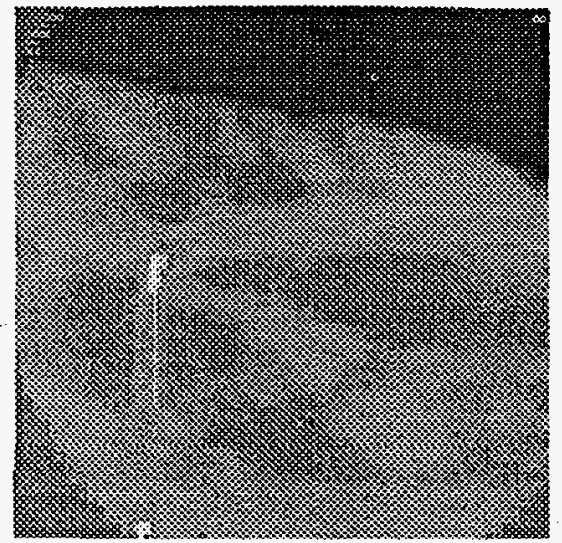

On SF6 $\left(941 \mathrm{~cm}^{-1}\right)$

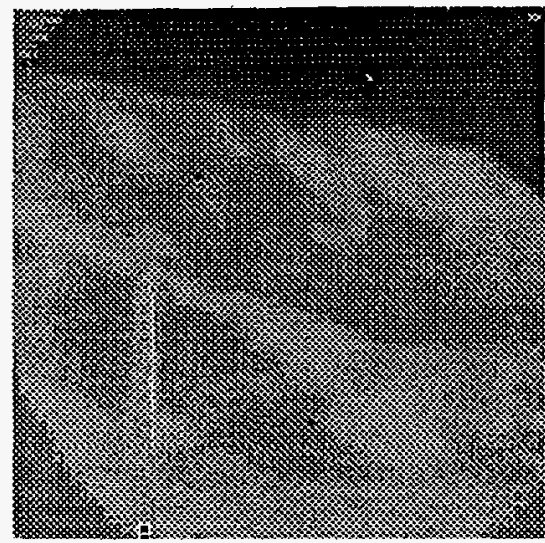

Off SF6 $\left(895 \mathrm{~cm}^{-1}\right)$

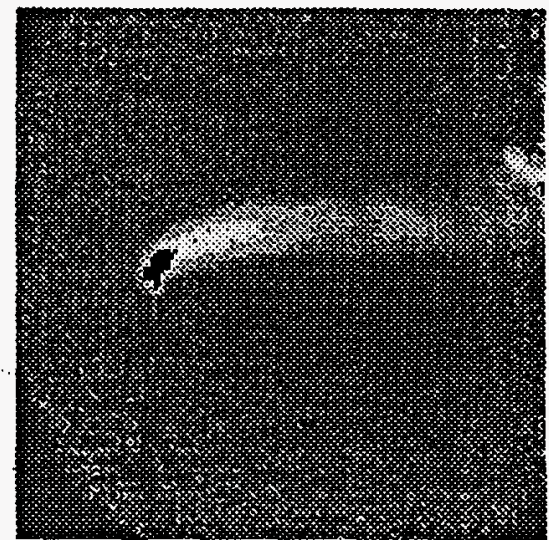

Difference

Figure 3. Hyperspectral imaging observations of a plume of $\mathrm{SF}_{6}$ with hills in the background are displayed. The left most image is a single spectral channel section of the data cube near the peak of the $\mathrm{SF}_{6}$ intensity. The middle image corresponds to a nearby spectral channel with no strength from $\mathrm{SF}_{6}$. Almost all of the structure in this scene, except for the location of the gaseous vapor, is due to temperature variations. Some of the structure in the difference image is the result, not of plume radiant intensity variations, but a varying degree of radiance contrast with respect to the background. Near the center of the image, for example, the difference intensity is noticeably smaller. This is because at this position in the image, the ground brightness temperature nearly equals the plume temperature.

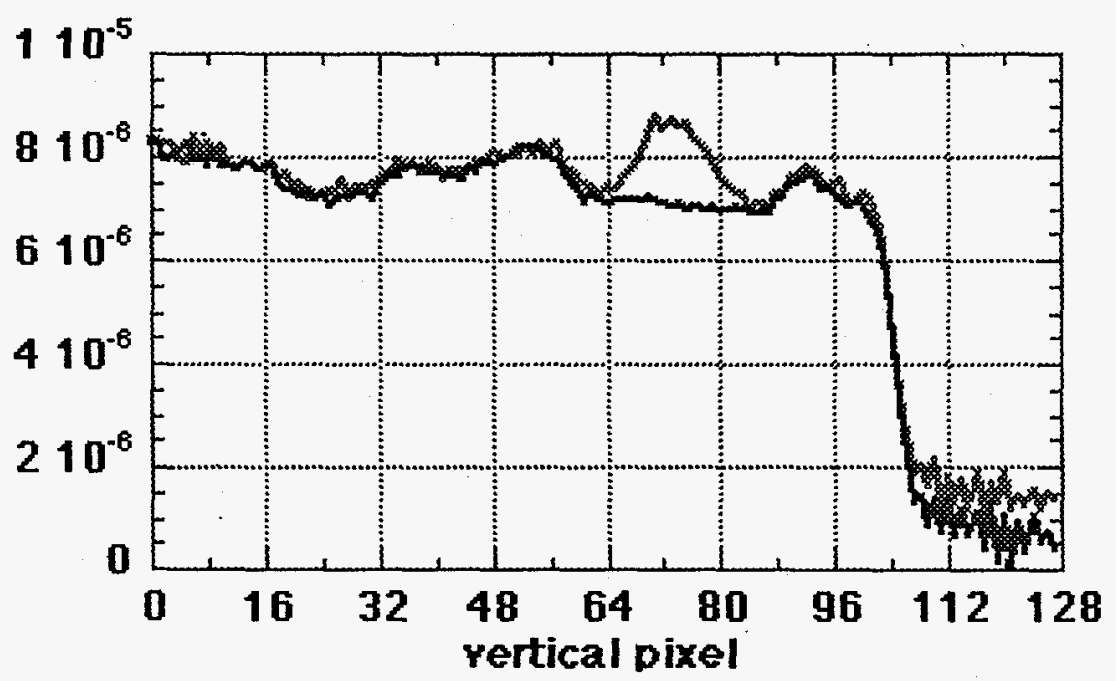

Figure 4. A plot of vertical line-outs of the calibrated radiant intensity in units of W/cm $/ \mathrm{sr}^{2} / \mathrm{cm}^{-1}$ are displayed. Both line-outs extend from the middle of the bottom of each image in figure 3 to the middle of the top of each image. The radiant intensity for the $895 \mathrm{~cm}^{-1}$ spectral channel has been scaled by a factor of 0.92 in order to remove the average Planck factor, and to emphasize the lack of "color" in the infrared images. The sharp drop in radiance near pixel 100 corresponds to the transition from hills to sky. 
In the images in figure 2, the gas plume was observed from below, with clear sky in the background. The thermal radiance from clear sky is quite low in the water window regions of the spectrum, and thus a high radiance contrast is obtained even for optically thin plumes at ambient air temperature. For observations with the plume viewed against the ground, there is still a significant radiance contrast between the plume and the background. Although the plume quickly equilibrates to the ambient air temperature after emerging from the source region, the ground temperature is almost always either warmer (in the sunlight) or colder (at night), than the ambient air temperature. On the average, there are only two "crossover" times during a 24 hour period when the ground temperature and the air temperature are nearly the same. At other times it is reasonable to expect approximately a $10^{\circ}$ temperature contrast between the ground and the air. An example of the infrared hyperspectral imaging of a release of $\mathrm{SF}_{6}$ as viewed with ground behind the plume is displayed in figure 3 . This data cube illustrates the advantage of hyperspectral imaging for the detection of weak spectral anomalies. In the image at the wavenumber corresponding to $S_{6}$, shown at the left in figure 3 , there is sufficient variation of the background radiance within the scene that it is quite difficult to observe the gas plume. By a careful comparison of the visual appearance of the left and middle images, it is possible, but somewhat difficult, to trace the general extent of the gas plume. However, by taking a difference between the two images, which corresponds to the excess radiance coming from the thermal emission of the plume, it becomes very easy to see the plume. It can also be observed in figure 3 that the difference image becomes very bright at the extreme right of the field of view, where the gas plume happens to extend above the horizon, and is then viewed against the much colder sky.

In figure 4, some quantitative results from this data cube are shown for a single vertical line-out taken through the middle of the images on and off the spectral peak of the plume emission. From these curves it is apparent that the magnitude of the background radiance variation is quite comparable to the magnitude of the excess thermal emission from the plume. The analysis of this data cube illustrates that it is quite possible to exploit the natural variability in scene radiance in order to observe gases which have equilibrated to the local air temperature, even if the average ground temperature is identical to the air temperature.

\section{TEMPORAL VARIATIONS}

One of the commonly expressed disadvantages of time multiplexed FTIR spectrometers is the problem of observing fluctuating sources. Any significant temporal fluctuations in the observed light intensity within the frequency range corresponding to the spectral features of interest represents noise in the derived spectrum. Even worse, periodic fluctuations produce line shifts of the observed spectral features. For remote sensing of natural phenomena, strictly periodic sources are not very common. Randomly fluctuating sources, however, are ubiquitous, and imaging FTIR spectroscopy might be thought hopeless in the presence of strong temporal fluctuations. It turns out not to be completely hopeless. It is possible, by using the squared magnitude of the Fourier transform of the interferogram, rather than simply the real part, to observe relatively simple structure in the portion of the "spectrum" which is produced by the temporal fluctuations of the source. An example of the effect of temporal fluctuations on the spectra measured with LIFTIRS is presented in figure 5. This figure displays the power spectrum from a representative group of pixels in the image of the rapidly fluctuating hot $\mathrm{CO}_{2}$ emission from a jet engine as observed at a distance of approximately $1 \mathrm{~km}$ with an $\operatorname{lnSb}$ focal plane array. The sensitivity of the InSb detector becomes vanishingly small below approximately 2,000 
$\mathrm{cm}^{-1}$. Any spectral intensity observed outside the pass band of the detector is obviously generated by the temporal fluctuations. In figure 5 , the system bandpass of the LIFTIRS instrument with an InSb array has been blanked out in order to emphasize the global behavior of the fluctuation spectrum. In the region displayed in figure 5 , the magnitude squared of the Fourier transform of the interferogram is precisely the power spectrum of the turbulent fluctuations of the infrared emission. The solid line fit displayed in this figure represents a $1 / f$ power law, and it can be seen to be an excellent representation of the shape of the observed distribution.

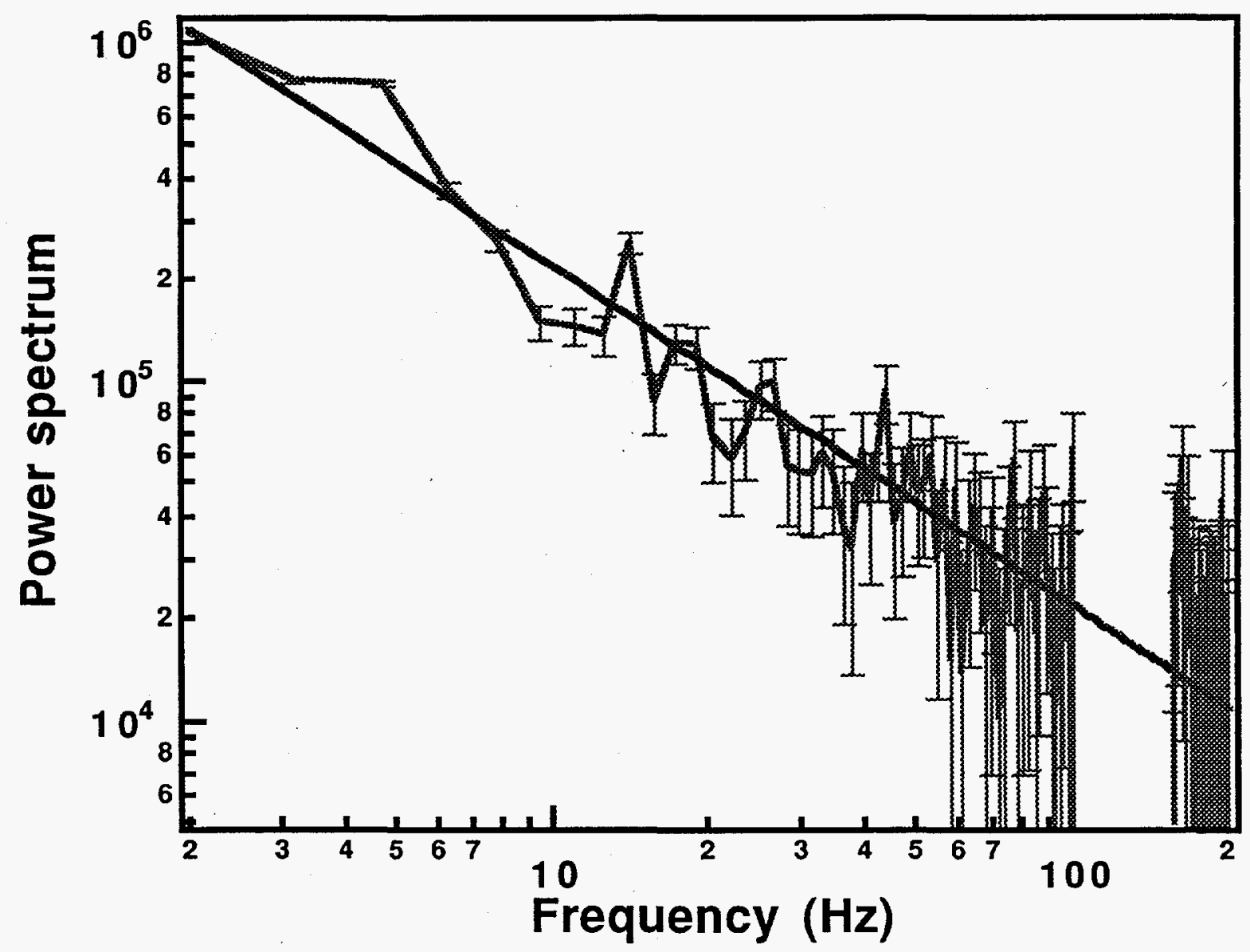

Figure 5. The fluctuation power spectrum produced by turbulent, hot gaseous emission from a jet engine is displayed. The solid line represents a $1 / f$ power law fit to the spectral intensity vs. frequency. As is often the case for fully developed turbulence, a simple $1 / f$ power law produces a very good fit to the data. The blank region between 100 and $160 \mathrm{~Hz}$ corresponds to the region of $\mathrm{CO}_{2}$ emission near $2300 \mathrm{~cm}^{-1}$.

Because of the simplicity of the power spectrum of the fluctuations, viz. $1 / f$, it requires only a single parameter to accurately fit it. It is thus easy to estimate the fluctuation generated background spectrum. In figure 6 , the net spectrum observed from the hot jet engine is displayed. In this figure, the absolute frequency in $\mathrm{Hz}$ has been converted into the equivalent 
wavenumber units. The size of the error bars shown in this plot is dominated by the uncertainty in the background subtraction.

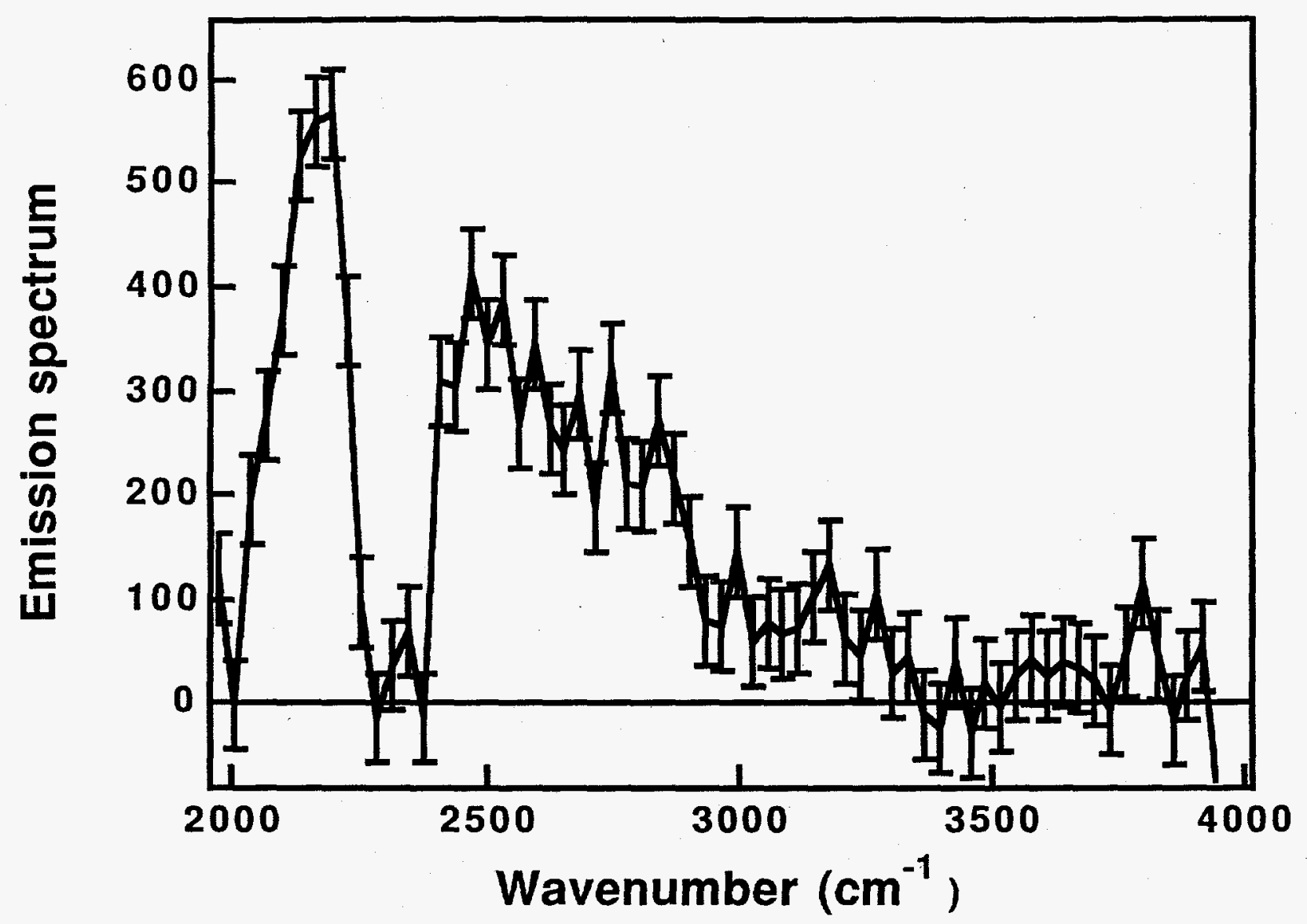

Figure 6. The net emission spectrum observed after subtraction of the underlying $1 / \mathrm{f}$ background is displayed.

The most significant aspect of the net spectrum displayed in figure 6 is that the errors generated by the uncertainty in subtraction of the intensely fluctuating background are only at the $10 \%$ level over much of the bandpass of the system. Another important point about the data displayed in figure 6 is that it was not necessary to specify, a priori, what the tradeoff between temporal resolution and spectral resolution was going to be before the measurements were made. In fact, a longer series of interferogram measurements was made than was used in the calculation of the spectrum displayed in figure 6 . The width of the interval about the centerburst utilized was varied to achieve the most favorable compromise between SNR, spectral resolution, and temporal resolution.

\section{CONCLUSIONS}

In summary, we have found that the flexibility of LIFTIRS in terms of readily variable spectral resolution and temporal resolution makes it very easy to adapt to different observational requirements. It is quite easy to choose, after the fact, which spatial region is of greatest interest, 
or which temporal interval is of greatest significance. It is also easy to diagnose the presence of temporal fluctuation generated noise sources by examination of the out of band noise levels. It is finally most convenient to use "through the spectrometer" imaging in order to properly aim at a subject of interest.

\section{ACKNOWLEDGMENTS}

This work was performed under the auspices of the U.S. Department of Energy under Contract No. W-7405-Eng-48*. We thank Mark Eckart for providing support for this effort. We also thank Johnathon Marks, Ron Robinson, Chuck Lee, Bill Aimonetti, Bill Kost, Dana Hargrove, and Don Western for their help in fielding the LIFTIRS instrument and acquiring the data.

\section{REFERENCES}

1. A.E. Potter, Jr., "Multispectral Imaging System", U.S. Patent \# 3702735, Nov. 14, 1972.

2. C.W. Wells, A.E. Potter, T.H. Morgan, "Near-infrared spectral imaging Michelson interferometer for astronomical applications", in the proceedings of the conference: Infrared Imaging Systems Technology, April 10-11, 1980, Washington, D.C., ed. J. Zimmerman, W.L. Wolfe, SPIE vol 226.

3. C.L. Bennett, M. Carter, D. Fields, J. Hernandez, "Imaging Fourier Transform Spectrometer", p.191-200, in the proceedings of the Imaging Spectrometry of the Terrestrial Environment, April 14-15, 1993, Orlando, FI., SPIE vol 1937.

4. M.R. Carter, C.L. Bennett, D.J. Fields, J. Hernandez, "Gaseous effluent monitoring and identification using an imaging Fourier transform spectrometer", p.16-26, in the proceedings of the Substance Detection Systems, October 5-8, 1993, Innsbruck, Austria, SPIE vol 2092.

5. M.R. Carter, C.L. Bennett, D.J. Fields, D. Lee, "Livermore Imaging Fourier Transform Infrared Spectrometer (LIFTIRS)", in the proceedings of Imaging Spectrometry, April 17-19, 1995, Orlando, FI., SPIE vol 2480.

6. Reinhard Beer, Remote Sensing by Fourier Transform Spectrometry, John Wiley \& Sons, Inc., NY, 1992.

* by Lawrence Livermore National Laboratory 